\title{
X-ray Fluorescence Analysis in an Electron Microscope: Improved Spotsize of Polycapillary Focusing Optics at the IfG Modular X-ray Source (iMOXS/2®)
}

\author{
Magnus Menzel and Aniouar Bjeoumikhov ${ }^{1}$ \\ 1. IfG - Institute for Scientific Instruments GmbH, Berlin, Germany.
}

The IfG modular X-ray source iMOXS $/ 2^{\circledR}$ can be attached to any scanning electron microscope (SEM) with a special adapter flange. Combining the iMOXS/2® with the existing energy dispersive X-ray detection system enables X-ray fluorescence analysis (XRF). This allows taking advantage of the high elemental sensitivity of XRF compared to electron probe microanalysis (EPMA) [1].

Guiding and focusing the X-rays from the X-ray tube within the iMOXS/2® to the sample is accomplished by using polycapillary optics. A multitude of glass capillaries with a diameter in the range of a few micrometers utilizes multiple total reflections to focus the X-rays [2]. This is a challenge, especially to achieve small spotsizes combined with a high photon flux, large output focal distance and a variety of source to sample distances (up to $500 \mathrm{~mm}$ ). Therefore, IfG constantly works on improving their polycapillaries.

In this work, an iMOXS/2® system attached to a Zeiss Evo 40 electron microscope was equipped with new generation of polycapillary optics. Test structures of $\mathrm{Cu}$ on a Si-wafer were examined with this setup. The test structure is moved with the $\mathrm{X}-\mathrm{Y}-\mathrm{Z}$ table of the electron microscope, while the iMOXS/2® illuminates a fixed, but adjustable, position. Figure 1 shows a test structure of $50 \mu \mathrm{m} \mathrm{Cu}$ stripes on a Si-wafer with increasing spacing in three steps from left to right of 50, 100 and $150 \mu \mathrm{m}$. An $\mathrm{XRF}$ linescan over that structure demonstrates, that the intensity of the $\mathrm{Cu} \mathrm{K \alpha}$ as well as the opposed $\mathrm{Si}$ $\mathrm{K} \alpha$ signal can clearly resolve the structure. This is true for all three different distances between the stripes.

While Figure 1 demonstrates the potential of the iMOXS/2® for linescans, Figure 2 presents the result of an XRF map. This is particularly interesting, since it demonstrates the $\mathrm{X}$ and $\mathrm{Y}$ dimension of the spot. Again the test structure is $\mathrm{Cu}$ on a wafer, this time $50 \times 50 \mu \mathrm{m}$ squares of $\mathrm{Cu}$ with $50 \mu \mathrm{m}$ spacing. The far right squares are reduced to $50 \times 25 \mu \mathrm{m}$. A map scan over the whole structure, enabled by the detector software, shows the squares are distinguishable from each other. This improved spatial resolution requires fine adjustment of the iMOXS/2® instrument, especially of the focus on the sample, and was only achieved with this new generation of polycapillary optics.

The improved focal spotsize of the polycapillary optics leads to an improved spatial resolution of the $\mathrm{XRF}$ analysis enabled by the $\mathrm{iMOXS} / 2{ }^{\circledR}$ instrument. This is particularly interesting if several small structures are to be analysed with trace amounts of heavy elements. Due to the high background using electron excitation caused by bremsstrahlung, detection limits of EPMA are usually in the range of 1-0.1 mass\%. XRF analysis improves this detection limit down to the range of $100 \mathrm{ppm}$ or even less.

References:

[1] M Procop, V-D Hodoroaba and V Rackwitz, Microscopy and Analysis 25 (2011), p. 11.

[2] A Bjeoumikhov, S Bjeoumikhova and R Wedell, Part. Part. Syst. Charact. 22 (2005), p. 384. 


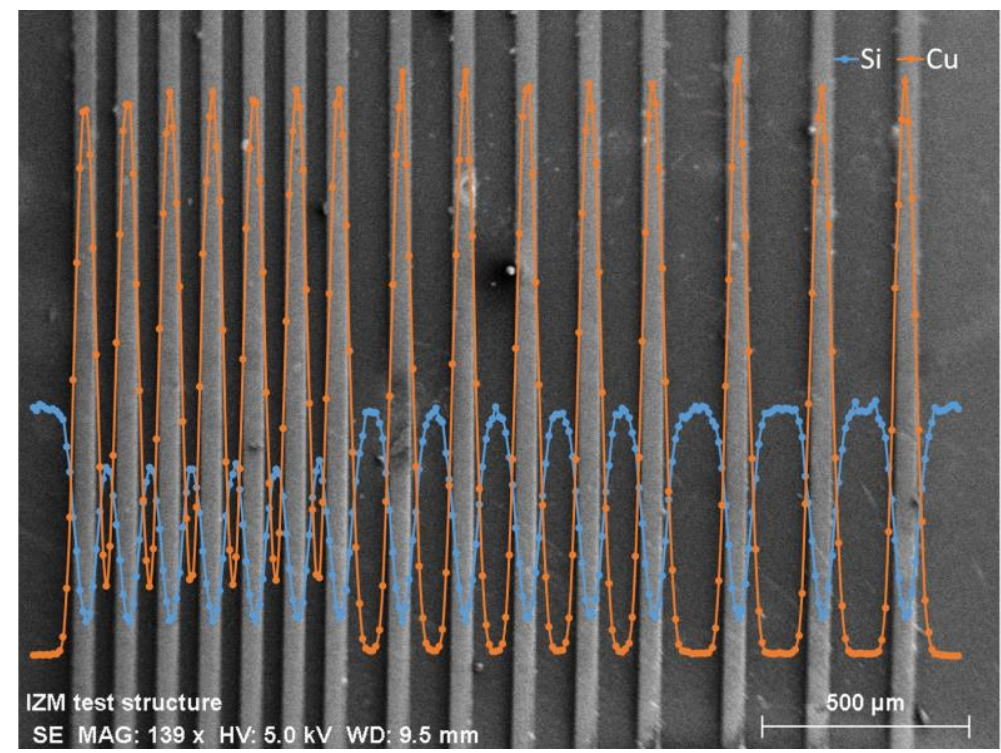

Figure 1. Results of an XRF linescan performed by moving the sample over a $\mathrm{Cu}$ structure on a $\mathrm{Si}$ wafer shown beneath (secondary electron picture).
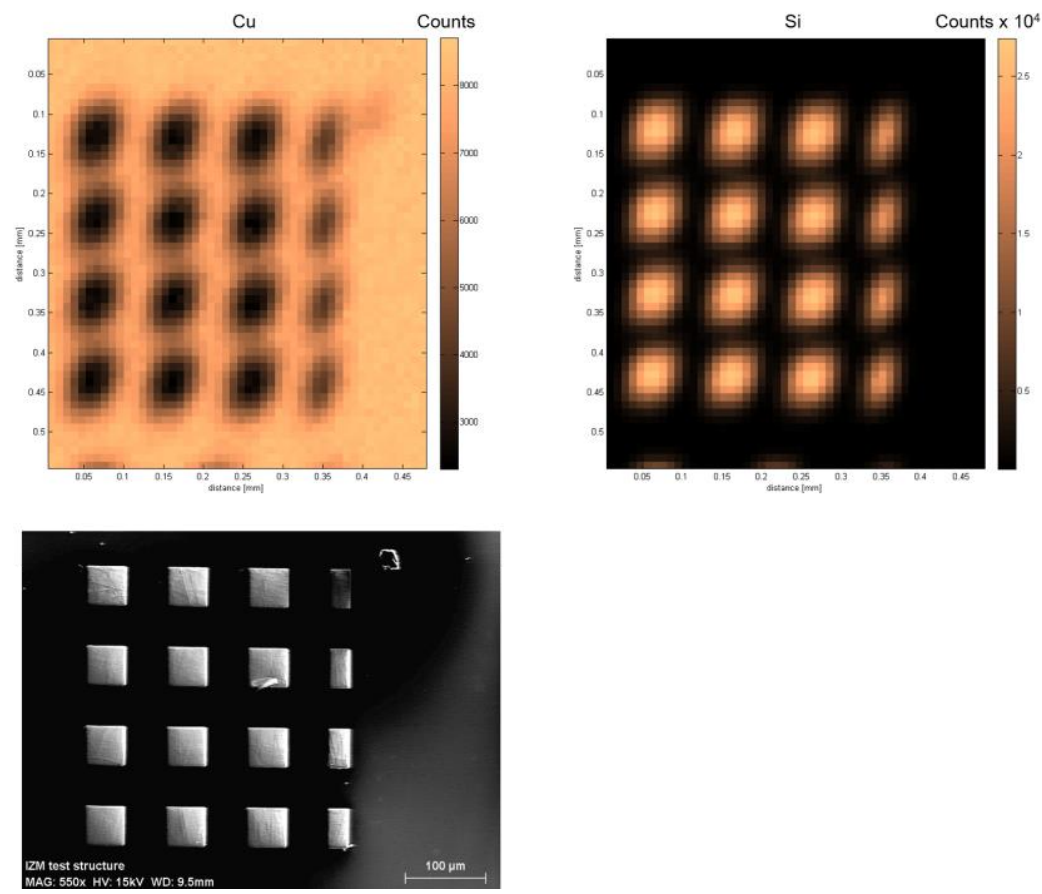

Figure 2. XRF Maps of $\mathrm{Cu} \mathrm{Ka}$ and $\mathrm{Si} \mathrm{Ka}$ (top left and top right), compared to the picture generated by the detection of secondary electrons. 